\title{
La Edad y el Sexo en la Orientación a la Comparación Social
}

\author{
The Age and Sex in the Social Comparison Orientation
}

\author{
Alfonso Urzúa M. \\ Patricia Zúñiga B. \\ Universidad Católica del Norte, Chile. \\ Abraham P. Buunk \\ Academia Real de Artes y Ciencias \& Universidad de Groningen, Netherlands.
}

(Rec: 16 de diciembre de 2011 / Acept: 19 de julio de 2012)

\begin{abstract}
Resumen
La Orientación a la Comparación Social (OCS) es la disposición personal de los individuos propensos a la comparación social. El objetivo de este estudio fue analizar la OCS en distintas edades en hombres y mujeres. La hipótesis principal es que la media en OCS es mayor en los rangos de edades menores y desciende a medida que aumenta la edad. Participaron 2757 personas, entre los 10 y 88 años de edad, de la ciudad de Antofagasta, que fueron evaluadas con el cuestionario INCOM - E. Los resultados indican una disminución significativa en la media de la OCS a medida que aumenta la edad de los rangos. Se encuentra también que la media en OCS es significativamente mayor en los hombres que en las mujeres. Los alcances de este estudio abren paso a nuevas líneas de investigación como las implicancias de las construcciones de género en la OCS. Palabras clave: orientación a la comparación social, edad, sexo.
\end{abstract}

\begin{abstract}
The Social Comparison Orientation (SCO) is the personal disposition of individuals prone to social comparison. The aim of this study was to analyze the SCO at different ages in men and women. The main hypothesis is that the average is higher in SCO younger age ranges and decreases as age increases. Participants 2757 people between 10 and 88 years of age in the city of Antofagasta, which were assessed with a questionnaire INCOM - E. The results show a significant decrease in the mean SCO with increasing age ranges. It is also found that the average SCO is significantly higher in men than in women. The scope of this study open up new lines of research and the implications of gender constructions in the SCO.

Keywords: social comparison orientation, age, gender.
\end{abstract}

\section{Introducción}

La comparación social como objeto de estudio ha sufrido numerosas transiciones y reformulaciones (Buunk \& Mussweiler, 2001; Suls, Martin \& Wheeler, 2002; Suls \& Wills, 1991). Inicialmente, el concepto se desarrolló a partir de un enfoque centrado principalmente en la autoevaluación en grupos. Posteriormente, se ha ampliado a diversos campos de investigación que abarcan muchas perspectivas y enfoques diferentes (Buunk \& Gibbons, 2006; Buunk, Ybema, Gibbons \& Ipenburg, 2001).

El proceso de comparación social fue propuesto inicialmente por Festinger (1954). Hace referencia a la comparación que realiza un sujeto de sus opiniones y habilidades con respecto a las de su grupo. Festinger señaló que necesitamos compararnos con nuestros iguales, tanto para evaluar nuestras opiniones y capacidades, como para obtener una validación por consenso de nuestras creencias y actitudes (Turner,1990). Hipotetizó que en los seres humanos existiría un impulso a evaluar sus opiniones y sus capacidades y que, en tanto no se disponga de medios objetivos no sociales, la gente evalúa sus opiniones y capacidades comparándolas con las opiniones y capacidades de otros (Buunk, Belmonte, Peiró, Zurriaga \& Gibbons, 2005; Buunk \& Gibbons, 2006; Tajfel, 1981).

Este artículo es producto de un estudio financiado por la Comisión Nacional de Investigación Científica y Tecnológica del Gobierno de Chile - CONICYT, a través del proyecto FONDECYT 11100090.

Correspondencia: Dr. Alfonso Urzúa M, Escuela de Psicología, Universidad Católica del Norte, Antofagasta (Chile), fono: 055355843, E-mail: alurzua@ ucn.cl 
Para Festinger, la gente estaría especialmente interesada en información concerniente a las opiniones y habilidades de sus similares, las que le permiten al individuo evaluar su propio ajuste a las nuevas situaciones. La similitud incrementa el valor de la información que se puede obtener a través de la comparación, en el sentido de que el individuo se compararía con personas que presenten similitud con él, en la dimensión en que se produce la evaluación, o con aquellas que se encuentren en un proceso de ajuste similar, o con las que se sienta en condiciones parecidas en algunas áreas, para luego compararse con ellas en la dimensión evaluada (Buunk, 2006; Gibbons \& Buunk, 1999; Taylor, Wood \& Lichtman, 1985). Ybema \& Buunk (1993) ampliaron las ideas originales de Festinger, señalando que el grado de identificación o similitud viene dado por la cercanía, o por considerar al modelo de la comparación como ejemplo del propio funcionamiento o expectativas en un futuro.

\section{Efectos de contraste e identificación}

Generalmente, se ha asumido que la comparación social tiene efectos de contraste, es decir, las personas que tienen tendencia a compararse con otros en mejor situación (comparaciones hacia arriba), tienen mayores efectos negativos en sus afectos (Ambrosio et al., 1991; Crosby, 1976; Major, 1994; Morse \& Gergen, 1970; Suls, 1986 en Buunk, Peiro, Rodríguez \& Bravo, 2007; Buunk, 2006). En cambio, aquellos que tienen la tendencia de compararse con otros en peor situación (comparaciones hacia abajo), pueden generar mayor afecto positivo, especialmente en individuos sometidos a estrés o a enfermedades (Buunk, 2006; Buunk \& Ybema, 1997; Gibbons, Gerrard, Lando \& McGovern, 1991; Taylor, Wood \& Lichtman, 1985 en Gibbons \& Buunk, 1999; Tennen, McKee, \& Affleck, 2000 en Friedswijk, Buunk, Steverink \& Slaets, 2004; Van der Zee, Buunk, \& Sanderman, 1998; Van Yperen, Brenninkmeijer \& Buunk, 2006; Wheeler \& Miyake, 1992).

Por otra parte, se han encontrado hallazgos sobre efectos de asimilación que puede tener la comparación social. Wheeler (1966) sugirió que los individuos pueden participar en comparaciones hacia arriba o hacia abajo, para confirmar que son similares a las comparaciones de meta o expectativas. Heider (1958) señaló que las personas en respuesta al destino de otra, pueden experimentar sentimientos concordantes con la suerte de otro. De esta forma, la comparación hacia abajo generaría sentimientos negativos, porque se experimenta el sufrimiento de otros, en cambio la comparación hacia arriba generaría una buena sensación, puesto que se disfrutaría de la suerte de otros (Brenninkmeijer \& Buunk, 2006; Buunk et al., 2005; Gómez - Jacinto, 2005; Suls, Martín \& Wheleer, 2000; Suls \& Wills, 1991; Van Yperen, Brenninkmeijer \& Buunk, 2006). En general, cuando se produce la asimilación, las comparaciones hacia arriba pueden evocar una variedad de respuestas positivas, como el optimismo, la admiración e inspiración, mientras que las comparaciones hacia abajo pueden provocar varias respuestas negativas, tales como la preocupación, piedad o vergüenza (Blanton, 2000; Buunk \& Ybema, 1997; Collins, 1996; Smith, 2000 en Buunk et al., 2005).

Muchos investigadores de la comparación social han señalado una reticencia de las personas a admitir que uno se compromete en comparaciones sociales (Brickman \& Bulman, 1977; Helgeson \& Taylor, 1993; Hemphill \& Lehman, 1991; Wood, 1996 en Buunk \& Gibbons, 1999; Buunk \& Mussweiler, 2001). Esto puede deberse, por un lado, a que admitir las comparaciones sociales no es algo socialmente deseable y, por otro, a que los individuos a menudo no son conscientes de las comparaciones que hacen. Por otra parte, parece posible que aunque todos los individuos se comparen a sí mismos con otros en ocasiones, muchos de ellos pueden, de hecho, hacerlo con poca frecuencia (Buunk et al., 2005).

\section{Diferencias individuales en la comparación social}

En cuanto a diferencias individuales en la comparación social, tenemos que, entre las variables de personalidad que influyen en este proceso está en primer lugar la autoestima, que parece jugar un papel destacado en particular y, como consecuencia, ha recibido la mayor atención empírica (Aspinwall \& Taylor, 1993; Gibbons \& McCoy, 1991; Wheeler \& Miyake, 1992 en Buunk \& Mussweilwer, 2001; Buunk, Collin, Taylor, VanYperen \& Dakof, 1990; Michinov, 2007; Wood, 1989). Otra variable de personalidad que ha sido abordada en el estudio de la comparación social, es el neuroticismo, que se considera como un factor de estrés predisposicional (Buunk, 2006; Van der Zee, Buunk \& Sanderman, 1998) en enfermedades como el cáncer, donde se asocia neuroticismo a un mayor interés en información (Van der Zee, Buunk \& Sanderman, 1998).

Otra línea investigativa en el estudio de la comparación social dice relación con importantes diferencias individuales en la medida en que las personas se comparan con otros. Se trataría de factores personales y psicosociales que modulan su frecuencia de uso. Este enfoque, que estudia la tendencia a compararse con los demás, ha sido llamado por Gibbons \& Buunk Orientación a la Comparación Social (OCS). Esta OCS es un concepto nuevo que reflejaría una disposición o tendencia personal a compararse con los demás, es decir, la orientación de los individuos que son propensos a la comparación, a tomar en cuenta los sentimientos y respuestas de los demás, y una inclinación bastante fuerte a acomodarse a los otros en circunstancias similares (Buunk et al., 2005; Gibbons \& Buunk, 1999; Michinov \& Michinov, 2001; Michinov, 2007; Terol et al., 2008).

Se ha visto que las personas difieren en la frecuencia de la comparación, en la tendencia a compararse con otros, en la sensibilidad a la información de la comparación social, y en la construcción cognitiva de la situación en la que se comparan (Buunk, 2006). Gibbons \& Buunk (1999) desarrollaron una escala de evaluación llamada INCOM, que mide las diferencias individuales en la tendencia a 
compararse con los demás. También, se desarrolló una versión española de la escala, denominada INCOM - E en el 2005, con una fiabilidad de .80 y una estructura factorial similar a la versión original. La escala INCOM ha demostrado reflejar el interés en la información acerca de los pensamientos, logros y comportamiento de los otros en circunstancias similares (Buunk et al., 2005; Buunk \& Mussweiler, 2001; Gibbons \& Buunk, 1999; Michinov \& Michinov, 2001). Un segundo hallazgo de la escala se refiere a que la OCS refleja un alto interés por el sí mismo. Las personas que puntúan alto en la INCOM tienen: (a) un self más activado, (b) un autoconcepto más dinámico, que varía en diferentes situaciones sociales, (c) son más susceptibles de influencia social, (d) poseen una autoestima más baja, (e) una fuerte orientación hacia los demás, y (f) están más interesados en la validación de los demás que en reducir la incertidumbre (Buunk \& Gibbons, 2006; Michinov \& Michinov, 2001). Los autores indicaron que la OCS se podría explicar por cuatro variables, que son: autoconciencia privada, neuroticismo, orientación interpersonal e inclinación a conformarse a las demandas y expectativas de los demás. Por tanto, quienes tienen una alta OCS serían individuos que presentarían un elevado neuroticismo, altos niveles de ansiedad, sensibilidad social cuando las comparaciones son hacia abajo, estilo de apego ambivalente y alta conciencia de sí mismo, preocupación por sus pensamientos y sentimientos, alta orientación a las relaciones interpersonales, alta consideración de las necesidades de los demás y sensibilidad a sus sentimientos y conductas (Buunk et al., 2005; Buunk \& Mussweiler, 2001; Gibbons \& Buunk, 1999; Michinov \& Michinov, 2001).

En los últimos años ha habido un incremento en las investigaciones en cuanto a la relación de la OCS con otras variables, en tanto frecuencia y efectos de la OCS (Buunk \& Gibbons, 2006; Buunk, 2006). Los investigadores han indicado que los estudios sobre las diferencias individuales en la OCS y las variables que pueden influir en ésta, son necesarios para el avance de esta línea investigativa (Buunk \& Gibbons, 2006; Gibbons \& Buunk, 1999; Vander Zee, Buunk Sanderman, 1996). A continuación se puede observar un cuadro que sintetiza las variables estudiadas en su relación con la OCS (ver tabla 1).

Tabla 1. Variables estudiadas en su relación con la Orientación a la Comparación Social.

\begin{tabular}{|c|c|}
\hline Variable & Autores \\
\hline Interés en la comparación social & $\begin{array}{l}\text { Buunk, 2006; Buunk, Belmonte, Peiró, Zurriaga \& Gibbons, 2005; Gibbons \& } \\
\text { Buunk, 1999; Van der Zee, Oldersma, Buunk \& Bos, } 1998 .\end{array}$ \\
\hline Incertidumbre del self y estrés & $\begin{array}{l}\text { Buunk, 2006; Buunk et al., 2005; Buunk, Zurriaga, González, Terol y López- } \\
\text { Roig, 2006; Terol et al.,2008; Van der Zee et al., } 1998 .\end{array}$ \\
\hline Comparación social & $\begin{array}{l}\text { Buunk \& Gibbons, 2006; Buunk, 2006; Michinov \& Michinov, 2001; Michinov, } \\
\text { 2007; Terol et al., 2008; Van der Zee et al., } 1998 \text {. }\end{array}$ \\
\hline Comparación ascendente y descendente & $\begin{array}{l}\text { Buunk, 2006; Buunk et al., 2005; Buunk et al., 2006; Terol et al., 2008; Buunk, } \\
\text { Zurriaga, González - Romá \& Subirats, 2003. }\end{array}$ \\
\hline Burnout & Buunk, Ybema, Van der Zee, Schaufeli \& Gibbons, 2001. \\
\hline Depresión & Buunk \& Brenninkmeijer, 2001. \\
\hline Afrontamiento & Buunk, 2006; Buunk \& Brenninkmeijer, 2001. \\
\hline Neuroticismo & $\begin{array}{l}\text { Buunk et al., 2005; Buunk, Van der Zee \& Van Ypereng, 2001; Van der Zee, } \\
\text { Oldersma, Buunk, \& Bos, } 1998 .\end{array}$ \\
\hline Self & Buunk et al., 2005; Buunk et al., 2001. \\
\hline Afectos positivos y negativos & Buunk, Zurriaga, Peiró, Nauta \& Gosalvez, 2005. \\
\hline $\begin{array}{l}\text { Situaciones competitivas con énfasis en los } \\
\text { resultados }\end{array}$ & Buunk et al., 2005; Buunk et al., 2005. \\
\hline Clima social de cooperación en el trabajo & Buunk et al., 2005. \\
\hline Justicia y conductas antisociales & Thau, Wittek \& Aquino, 2007. \\
\hline Salud y conductas de riesgo & Gerrard, Gibbons, Reis - Bergan, Trudeau, Vande Lune \& Buunk, 2002. \\
\hline Satisfacción en las relaciones de pareja & Buunk, 2006. \\
\hline Cultura, género y self & Guimond et al., 2007. \\
\hline
\end{tabular}


En general, en las investigaciones sobre OCS no se han encontrado reportes sobre el comportamiento de la OCS en distintas edades, ya que estos han sido realizados fundamentalmente en adultos, por tanto no se tiene una visión de cómo se comporta la OCS en distintas edades y si existen además diferencias en la OCS según sexo en las distintas edades.

\section{Edad y orientación a la Comparación Social}

Aun cuando no se han reportado investigaciones sobre OCS en distintos rangos de edad, dado que se ha encontrado que la OCS se comporta de forma similar que la comparación social (Gibbons \& Buunk, 1999), se esperaría que ésta disminuyera a lo largo de la vida, tal como se señala en un estudio con pacientes crónicos, donde se observó que a mayor edad, menor era la OCS (Terol et al., 2008).

Es esperable que la OCS como proceso psicológico, en tanto se asemeja a la comparación social, se comporte de manera similar a ésta en las diversas etapas que atraviesa la vida de las personas. En las etapas de la infancia y adolescencia, dado el proceso de identificación social, la comparación social cumple un rol fundamental en la socialización. En estas etapas, las personas hacen mayor uso de este proceso, como forma de compararse e identificarse con otros que poseen características similares. Esta comparación opera de forma que los niños y niñas se comparan con los pares, tanto en rendimiento académico, entendiendo éste hacer bien o mal algo, como en juegos o competencias, sobre todo en el ámbito escolar. En la etapa de la pre adolescencia y adolescencia, este proceso se convierte en predictor de comparación con sus compañeros, en los ámbitos deportivo y social, puesto que se consolida pertenecer a un grupo determinado y se espera recibir valoraciones positivas por parte de sus pares (Huerta y Arduras, 2004). En la adolescencia y etapa juvenil, el proceso de comparación social se va modificando, puesto que domina un grupo reducido de pares y se produce el proceso de diferenciación con los otros y con la autoridad, por tanto las comparaciones se orientarían en tener determinadas características valoradas como superiores por él y la adolescente. El proceso opera en la imagen corporal en el caso de las mujeres en los patrones de belleza establecidos culturalmente y en el caso de los hombres en cultivar su cuerpo (musculatura) y aumentar el rendimiento deportivov(Jones, 2001; Wigfield, Eccles, Mac Iver, Reuman \& Migdley, 1991), y también se pueden ver sus efectos en el ámbito educacional, por la estructura de la educación secundaria, en que se favorece un formato más competitivo, en el cual se privilegia a los mejores (Huerta y Arduras, 2004; Wigfield et al., 1991). En cuanto a las etapas de adultez temprana y media, las personas son más seguras de su estado actual por tanto las comparaciones sociales son menos relevantes. Otro punto a mencionar es que la mayoría de los estudios están hechos considerando esos rangos de edades, y la edad no ha demostrado ser una variable a considerar aisladamente en los estudios, puesto que el proceso de comparación social se da con uno mismo y son otras variables psicológicas las que influyen en el momento en que se produce el proceso de comparación, lo mismo sucede con la OCS que correlacionó débil y negativamente con la edad (Buunk et al., 2005).

\section{Sexo y Orientación a la Comparación Social}

En cuanto a sexo, los autores reportaron que, por lo general, no encontraron diferencias en función de éste; no obstante, cuando tales diferencias se producían, las mujeres presentan mayor OCS en comparación con los hombres en estudios en los Estados Unidos y Los Países Bajos (Gibbons \& Buunk, 1999). En la muestra española, también se reportó que las mujeres mostraron una OCS más alta que los hombres (Buunk et al., 2005; Guimond et al., 2007). Posteriormente, en un estudio que incluye la variable género, se reportan diferencias entre los sexos en la OCS, y se señala que esta diferencia se debe principalmente a los prototipos de género que existen en la mayor parte de las culturas entre hombres y mujeres (Guimond et al., 2007), y podríamos decir que en nuestra cultura se debe a las desigualdades de género existentes, sobre todo en América Latina, que se traducen en nudos críticos entre las relaciones de género y otros vínculos de discriminación, sobre todo en las mujeres (Milosavljevic, 2007). En general, los resultados sugieren que, en consonancia con investigaciones previas, los estereotipos de género son similares en todas las culturas. Lo que diferencia y da cuenta de las variaciones en las diferencias de género es el grado en que las mujeres y los hombres utilizan el estereotipo de su propio grupo para definirse a sí mismos, resultado impulsado en gran medida por los procesos de comparación social (Guimond, Chatard, Martinot, Crisp \& Redersdorff, 2006; Guimond et al., 2007).

Considerando que los procesos cognitivos van modificándose a medida que el ser humano atraviesa por las distintas etapas de su vida, es decir, a medida que aumentan la edad, es posible hipotetizar entonces que la media en OCS será mayor en los rangos de edades menores y que descienda a medida que aumenta la edad (Terol et al., 2008; Urzúa, et al., 2010), y como segunda hipótesis que la media en OCS es mayor en mujeres que en hombres (Buunk et al., 2005).

Bajo este considerando, la siguiente investigación tuvo como objetivo general analizar la OCS en distintas edades en hombres y mujeres. Y como objetivos específicos evaluar las posibles diferencias dadas por la edad en la OCS y evaluar las posibles diferencias dadas por el sexo en la OCS.

\section{Método}

La investigación responde a un enfoque cuantitativo. El tipo de diseño es no experimental, transversal, de tipo descriptivo-correlacional. 


\section{Participantes}

La muestra final estuvo constituida por 2752 personas entre los 10 y 88 años de edad, de la ciudad de Antofagasta. 1343 participantes son hombres $(49 \%)$ y 1414 mujeres $(51 \%)$, con una media de edad de $31,3(D E=20.01)$. Dada la amplitud del rango de edad observado, para efectos de análisis se optó por establecer rangos de edad de 10 años. Se definen así rangos de edad de 10 a 19 años, 20 a 29 años, 30 a 39 años, 40 a 49 años, 50 a 59 años, 60 a 69 años y más.

Para fines de este estudio no se registraron variables como nivel socioeconómico, estado civil o escolaridad (ver tabla 2).

Tabla 2. Descripción de los participantes por rango de edady sexo.

\begin{tabular}{lcccccc}
\hline \multirow{2}{*}{ Descriptivos } & \multicolumn{6}{c}{ Rango de edad en años } \\
\cline { 2 - 7 } & $10-19$ & $20-29$ & $30-39$ & $40-49$ & $50-59$ & $60 \mathrm{y}+$ \\
\hline \multirow{3}{*}{ Hombres } & 576 & 239 & $13)$ & $(4)$ & $(5)$ & $(6)$ \\
Mujeres & 622 & 224 & 123 & 131 & 87 & 184 \\
Total & 1198 & 463 & 233 & 242 & 185 & 355 \\
\hline
\end{tabular}

\section{Instrumento}

La Escala de Orientación Hacia la Comparación Social en su versión en español INCOM - E (Buunk et al., 2005), desarrollada por Gibbons \& Buunk (1999) denominada INCOM, evalúa las diferencias individuales en la tendencia a compararse con los demás, lo que denominaron OCS. La escala original está compuesta por 11 ítems, presentando datos de 22 muestras, en las cuales los coeficientes alfas de fiabilidad variaron de .77 a .85. La escala es bidimensional y mostró, en un análisis factorial, que una solución de dos factores se ajustaba a los datos bastante bien, con un factor relacionado con la comparación de aptitudes (cuán bien uno está actuando), y un factor relacionado con la comparación de opiniones (qué está uno pensando y sintiendo). El primer factor consta de 6 ítems, referidos a la posición de la comparación propia con los demás en varias dimensiones (llamada "aptitud" por Gibbons \& Buunk, 1999), con un valor propio de 4.07 , explicando el $37 \%$ de la varianza, y un segundo factor que contiene 5 ítems, referidos a la necesidad de comparar los propios sentimientos y pensamientos (etiquetado como "opinión” por Gibbons y Buunk, 1999), con un valor propio de 1.50 , explicando el $14 \%$ de la varianza. En el análisis factorial confirmatorio, la correlación entre los dos factores fue muy alta, de .79 , lo que señala que, aunque la INCOM se compone de dos factores que se distinguen bien, los factores están muy relacionados. Por eso, se recomienda no usar los factores independientemente. En esta muestra utilizamos la INCOM - E (Buunk et al., 2005), con un coeficiente de fiabilidad de .82, obtenida de una muestra de 449 estudiantes de pre - grado, de diferentes carreras de la Universidad Católica del Norte el año 2009
(Urzúa, Castillo, Gemmel y Campos, 2010). La escala consta de 11 ítems, con respuesta en formato likert de 5 puntos. Las respuestas varían desde " $1=$ totalmente en desacuerdo" hasta " $5=$ totalmente de acuerdo". Ejemplos de ítem serían: "Siempre me gusta saber lo que harían otras personas en mi lugar" $y$ "No soy una persona que se compara a menudo con los demás". El coeficiente de fiabilidad en esta muestra es de .82 .

\section{Procedimiento}

Una vez obtenida la aprobación del Comité de Ética de la Universidad Católica del Norte, se procedió a conseguir los permisos y coordinaciones correspondientes con los participantes, previa firma de un consentimiento informado. Los datos se recogieron entre los meses de abril y agosto del 2011, en diferentes consultorios e instituciones públicas, de salud y educacionales de la ciudad de Antofagasta. En el caso de las y los menores de edad se envió el formulario de consentimiento informado a sus padres o tutores. La aplicación del instrumento fue individual. El tiempo promedio para completar el INCOM-E fue de 5 minutos. Se aplicaron 2752 encuestas y se respondieron satisfactoriamente 2752 . No se descartaron cuestionarios durante el proceso. Se consideraron como criterios de exclusión la discapacidad cognitiva o visual, que dificultara la correcta cumplimentación del instrumento. Los análisis fueron realizados a través del programa estadístico SPSS. 17. Se utilizó la prueba t de Student para verificar diferencias de medias en la OCS entre hombres y mujeres y ANOVA factorial para analizar las diferencias por rango de edad y si existía interacción entre edad y sexo.

\section{Resultados}

\section{Orientación a la comparación social}

Tal como se muestra en la Tabla 3, los puntajes a nivel de la muestra total oscilaron entre los 11 y los 55 puntos. La media reportada por los hombres es significativamente mayor que la de las mujeres $(F(1,11)=7,359 ; p<.05)$. Lo que nos indica que, al contrario de lo mencionado en la literatura y en nuestra hipótesis, los hombres presentan una mayor media en la OCS que las mujeres (ver tabla 3 ).

Tabla 3. Medias en Orientación a la Comparación Social por sexo.

\begin{tabular}{lccc}
\hline & & \multicolumn{2}{c}{ Sexo } \\
\cline { 3 - 4 } Descriptivos & Muestra total & Hombres & Mujeres \\
\hline $\mathrm{n}$ & 2676 & 1305 & 1371 \\
Media OCS & 30.51 & 31.00 & 30.05 \\
$\mathrm{DE}$ & 8.77 & 8.94 & 8.58 \\
Ptje. Mínimo OCS & 11 & 11 & 11 \\
Ptje. Máximo OCS & 55 & 55 & 54 \\
\hline
\end{tabular}


La diferencia entre las medias de los distintos rangos es estadísticamente significativa $(F(5,2670)=13,63 ; p<.05)$, en donde la media del rango de 10 a 19 años es superior a todos los otros rangos, siendo esta diferencia estadísticamente significativa en la comparación con todos los grupos (ver tabla 4 y Figura 1, respectivamente).

Tabla 4. Medias en OCS por rango de edad.

\begin{tabular}{lcccccc}
\hline & \multicolumn{6}{c}{ Rango de edad en años } \\
\cline { 2 - 7 } Descriptivos & $10-19$ & $20-29$ & $30-39$ & $40-49$ & $50-59$ & $60 \mathrm{y}+$ \\
\cline { 2 - 7 } & $(1)$ & $(2)$ & $(3)$ & $(4)$ & $(5)$ & $(6)$ \\
\hline $\mathrm{n}$ & 1198 & 463 & 233 & 242 & 185 & 355 \\
Media OCS & $\begin{array}{c}31.90^{*} \\
\text { a.b,c,d,e }\end{array}$ & 30.09 & 28.57 & 28.38 & 28.58 & 30.09 \\
DE & 8,12 & 8,76 & 8,66 & 8,74 & 9,50 & 9,76 \\
$\begin{array}{l}\text { Ptje. Mínimo } \\
\text { OCS }\end{array}$ & 11 & 11 & 11 & 11 & 11 & 11 \\
$\begin{array}{l}\text { Ptje. Máximo } \\
\text { OCS }\end{array}$ & 54 & 55 & 50 & 51 & 55 & 54 \\
\hline
\end{tabular}

$*_{\mathrm{a}}=$ significativamente mayor que rango (2) $p<.05$

$* \mathrm{~b}=$ significativamente mayor que rango (3) $p>.05$

$* \mathrm{c}=$ significativamente mayor que rango (4) $p<.05$

$* \mathrm{~d}=$ significativamente mayor que rango $(5) p<.05$

$*_{\mathrm{e}}=$ significativamente mayor que rango $(6) p<.05$

Al observar el comportamiento de la media en OCS en los distintos tramos de edad, pero separando por sexo, se encuentra que en los hombres, con excepción del grupo de 60 y más años, se produce una disminución del puntaje a medida que aumenta la edad (ver Figura 2).

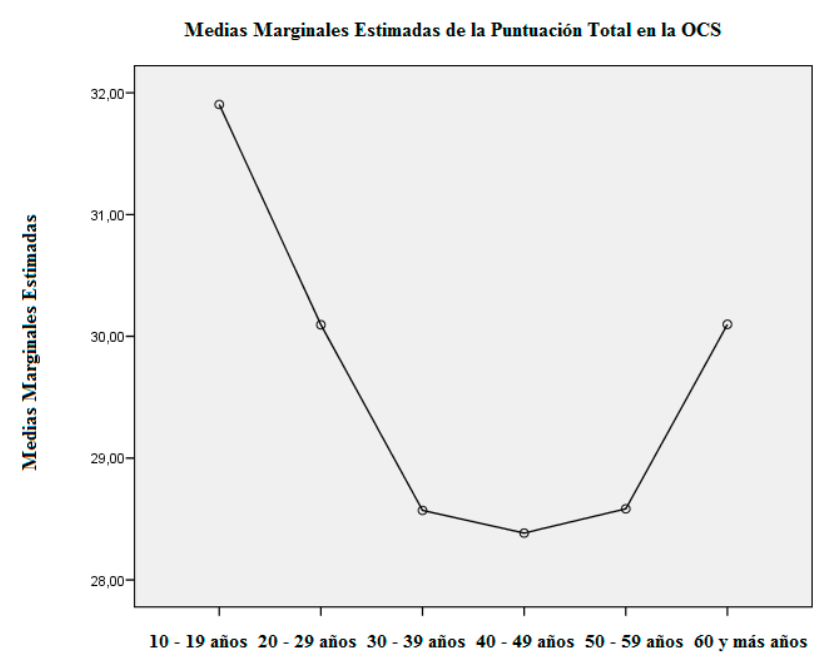

Figura 1. Medias en puntaje total OCS por rango de edad.

Las medias en OCS por tramo de edad y por sexo pueden ser observadas en la Tabla 5. Cabe destacar que la interacción entre sexo y edad no es estadísticamente significativa.

En los hombres, la diferencia entre los grupos es estadísticamente significativa $(F(5,1299)=7,359 ; p<.01)$. Con las comparaciones múltiples se pone en evidencia que la media de los participantes del rango de edad entre $10 \mathrm{y}$ 19 años es significativamente mayor que la de los rangos de 30 a $39(p<.05), 40$ a $49(p<.01)$ y 50 a 59 años $(p<.5)$. La media del rango de 20 a 29 años es significativamente mayor que el de 40 a 49 años $(p<.05)$ y que la del grupo de 50 a 59 años $(p<.05)$ (ver tabla 5$)$.

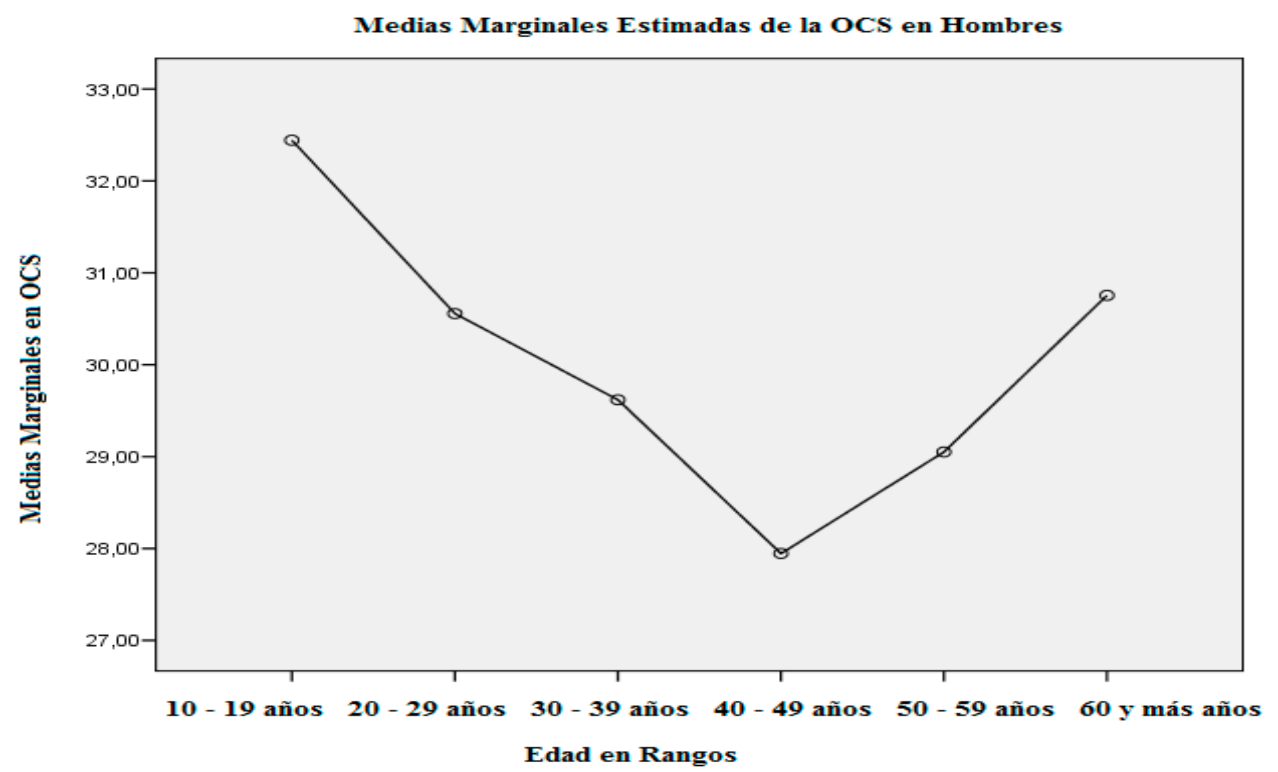

Figura 2. Medias en puntaje total OCS por rango de edad y sexo (Hombre). 
Tabla 5. Medias en Orientación a la Comparación Social por rango de edad y por sexo.

\begin{tabular}{|c|c|c|c|c|c|c|c|c|c|c|c|c|}
\hline & \multicolumn{12}{|c|}{ Rango de edad en años } \\
\hline & \multicolumn{2}{|c|}{$10-19$} & \multicolumn{2}{|c|}{$20-29$} & \multicolumn{2}{|c|}{$30-39$} & \multicolumn{2}{|c|}{$40-49$} & \multicolumn{2}{|c|}{$50-59$} & \multicolumn{2}{|c|}{$60 y+$} \\
\hline & \multicolumn{2}{|c|}{ (1) } & \multicolumn{2}{|c|}{ (2) } & \multicolumn{2}{|c|}{ (3) } & \multicolumn{2}{|c|}{ (4) } & \multicolumn{2}{|c|}{ (5) } & \multicolumn{2}{|c|}{ (6) } \\
\hline & $\mathrm{H}$ & $\mathrm{M}$ & $\mathrm{H}$ & M & $\mathrm{H}$ & M & $\mathrm{H}$ & M & $\mathrm{H}$ & $\mathrm{M}$ & $\mathrm{H}$ & $\mathrm{M}$ \\
\hline $\mathrm{n}$ & 576 & 622 & 239 & 224 & 110 & 123 & 111 & 131 & 98 & 87 & 171 & 184 \\
\hline $\mathrm{M}$ & 32.44 & 31.40 & 30.55 & 29.60 & 29.61 & 27.63 & 27.94 & 28.75 & 29.05 & 28.05 & 30.75 & 29.48 \\
\hline $\mathrm{ME}$ & 8.34 & 7.88 & 8.52 & 9.00 & 8.60 & 8.65 & 9.39 & 8.16 & 10.07 & 8.84 & 9.83 & 9.68 \\
\hline Mín & 11 & 11 & 11 & 11 & 11 & 12 & 11 & 12 & 11 & 11 & 11 & 11 \\
\hline Máx & 53 & 54 & 55 & 53 & 50 & 45 & 51 & 50 & 55 & 48 & 54 & 51 \\
\hline
\end{tabular}

En las mujeres también se encuentran diferencias estadísticamente significativas entre los rangos de edad $(F(5,1365)=7,015 ; p<.01)$, siendo la media del rango de 10 a 19 años significativamente mayor que la de los rangos de 30 a 39 años $(p<.05), 40$ a 49 años $(p<.05)$ y 50 a 59 años $(p<.05)$. La media del rango de 20 a 29 años es mayor que la de las mujeres de 40 a 49 años $(p<.05)$.

$\mathrm{Al}$ observar el comportamiento de la media en OCS en los distintos tramos de edad, pero separando por sexo, se encuentra que en las mujeres, con excepción del grupo de 60 y más años, se produce una disminución del puntaje a medida que aumenta la edad. Se puede observar un pequeño quiebre en la tendencia en el rango de 40 a 49 años, pero no es una diferencia significativa (ver Figura 3).

\section{Discusión}

En esta investigación se esperaba encontrar que la media en OCS fuese mayor en los rangos de edades menores, pero

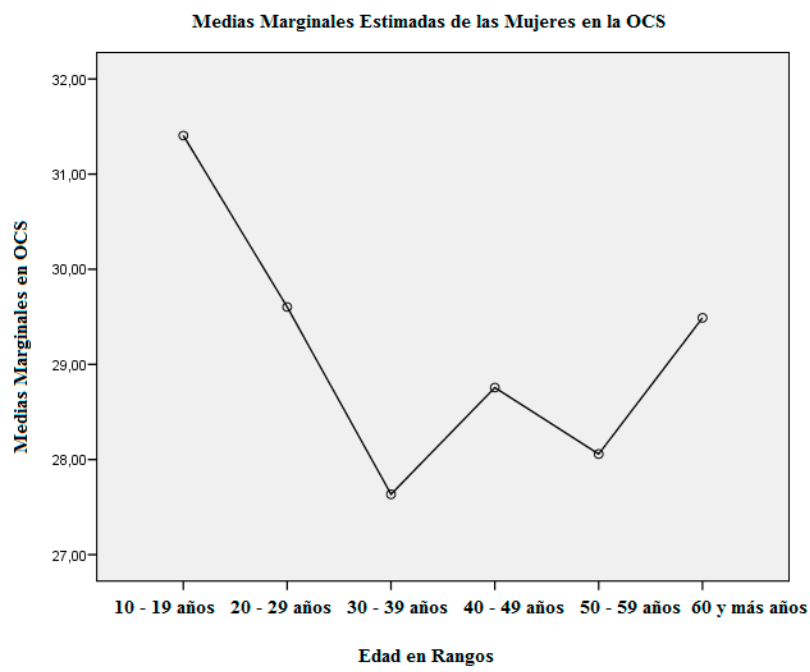

Figura 3. Medias en puntaje total OCS por rango de edad y sexo (Mujer). desciende a medida que aumenta la edad. Los resultados aportan evidencia para aceptar dicha hipótesis, toda vez que la media en la OCS es significativamente mayor en los rangos que comprende las edades de 10 a 19 años con relación a los grupos de mayor edad. Es posible que la OCS, al igual que la comparación social, se presente con mayor fuerza durante la infancia, adolescencia y la etapa juvenil, principalmente debido al proceso de identificación social, en el cual la comparación social cumple un rol fundamental en la socialización (Huerta y Arduras, 2004).

En este estudio, niños y niñas tendrían una mayor tendencia hacia la OCS, posiblemente asociado al compararse con los pares en diversas áreas, como rendimiento académico, juegos o competencias. En la etapa de la pre adolescencia y adolescencia, se puede observar que este proceso se convierte en predictor de comparación con sus compañeros en distintos ámbitos, principalmente el deportivo y social, esperando recibir valoraciones positivas por parte de sus pares (Huerta y Arduras, 2004). Por tanto, el mecanismo de compararse con los otros con características similares, como lo plantea Festinger (1954), en esta etapa cumple un rol fundamental y por esto se puede observar un incremento significativo en las diferencias respecto a otros rangos etarios.

Asimismo, las y los adolescentes que se encuentran en el rango de edad de 10 a 19 años, tendrían una mayor tendencia a la OCS, ya que al igual que en el caso anterior, la OCS podría modular el efecto de la comparación, toda vez que en estas edades la comparación social es parte del proceso de consolidación de la propia identidad y de diferenciación de los demás, como parte de una etapa de cambios físicos, biológicos, sociales, emocionales, en el sí mismo y en las relaciones con los demás. Existen dos factores que juegan un rol importante en los procesos de identificación y comparación social, que serían la imagen corporal y el rendimiento académico. La imagen corporal en los hombres se manifiesta de manera más competitiva, puesto que están más interesados en el rendimiento deportivo y cultivar un mejor cuerpo en el caso de las mujeres; en el atractivo físico. En el ámbito educacional, tanto en hombres y en mujeres 
se favorece un formato más competitivo por la estructura de la educación secundaria, en la que se privilegia a los mejores (Huerta y Arduras, 2004; Jones, 2001; Wigfield et al., 1991). Por tanto, quienes tienen una mayor tendencia a la OCS, generalmente la van a utilizar para mantener esa imagen personal superior, prefiriendo información congruente con esa imagen y rechazando actividades que la pongan en riesgo.

En el caso del grupo que corresponde al rango de 60 años y más, su media en OCS es mayor que los grupos de 20 a 29 hasta los de 50 a 59, interrumpiendo la tendencia a la baja que reporta las medias. Se ha reportado que tanto la OCS como la comparación ascendente de contraste disminuyen con la edad, sobre todo en la vejez, puesto que afectan negativamente a la percepción de calidad de vida (Taylor \& Lobel, 1989; Terol et al., 2008). Esto posiblemente se puede deber a factores que tienen relación con otras variables psicosociales, que se deberán aislar y estudiar en futuras investigaciones, como amenazas percibidas en el entorno, percepción de riesgo o pérdida, pasar por un estado elevado de estrés, de fragilidad y vulnerabilidad por la pérdida de funciones que produce la vejez, o utilizar la comparación con los demás para evaluar sus perspectivas frente al fin de la vida, entre otras, como lo señalan en estudios sobre comparación social y satisfacción con la vida (Gibbons, Gerrard, Lando \& McGovern, 1991; Frieswijk, Buunk, Steverink \& Slaets, 2004). Los factores descritos parecieran regular una mayor OCS en este grupo de edad, primero con la finalidad de regular las emociones negativas como consecuencia de la amenaza o pérdida, segundo para aumentar la sensación de bienestar tendiendo a realizar más comparaciones a la baja y aumentando la tendencia hacia la comparación social (Frieswijk et al., 2004; Taylor, Wood \& Lichtman, 1985; Ybema \& Buunk, 1995). Y tercero, como lo señala Taylor y Lobel (1989), que puedan utilizar indistintamente la comparación social ascendente y descendente para cubrir cualquier necesidad, con la finalidad de regular las emociones negativas y tener mejor calidad de vida (Van der Zee, Buunk, Sanderman, Botke \& Van den Bergh, 2000).

En cuanto a diferencias entre hombres y mujeres, se rechaza la hipótesis de investigación que señala que la media en OCS es mayor en mujeres que en hombres, puesto que los hombres de esta investigación reportaron diferencias significativamente mayores que las mujeres, a diferencia de lo que sugieren las investigaciones anteriores (Buunk et al., 2005; Guimond et al., 2007).

Esto posiblemente pueda tener explicación no tanto en el sexo biológico como tal, sino en las construcciones culturales vinculadas y construidas en torno a éste. Las diferencias de género están presentes en el medio social y cultural en que están insertos los participantes de nuestro estudio. Antofagasta, al ser una ciudad expuesta al fuerte influjo de la actividad minera, predominando el trabajo por turnos o de las largas ausencias del trabajador minero, más la base patriarcal que se encuentra en la cultura, muestra características que marcarían la vida socio relacional y afectivas de sus habitantes (Silva y Barrientos, 2008). También podríamos señalar como un factor, relacionado a lo expuesto con anterioridad, la noción de masculinidad, que se percibe en una imagen prototípica en que los hombres se ubican a una gran distancia de sus familias, descansan mucho en sus redes sociales primarias de apoyo (compañeros de faena), bajo fuertes demandas físicas, en un trabajo que es mirado como arriesgado para su salud (Barrientos, 2006). Ambos factores podrían explicar el comportamiento de la OCS en los hombres, ya que, en esta dinámica, serían más competitivos y por ende, presentarían una mayor tendencia a la OCS, puesto que por el contexto sociocultural de la ciudad, el rol de género de los hombres se validaría desde la obtención de recursos económicos y de un determinado estatus. Lo que finalmente da cuenta de que las variaciones en las diferencias de género se va a dar por el grado en que las mujeres y los hombres utilizan el estereotipo de su propio grupo para definirse a sí mismos, resultado impulsado en gran medida por los procesos de comparación social (Guimond et al., 2006; Guimond et al., 2007).

Podemos concluir que la OCS se comporta de manera distinta en los rangos de edad utilizados en nuestro estudio, mostrando la tendencia a ser menor a medida que aumentan en año los rangos etarios, siendo mayor en las etapas de infancia, adolescencia y juvenil, y presentando un descenso en las edades que comprenden la adultez, con excepción del rango 60 años y más, que presenta un aumento en comparación con los rangos antes mencionados. En cuanto a las diferencias en sexo, se encontró que sí existen diferencias significativas en hombres y mujeres, siendo el grupo de los hombres los que presentan mayor OCS.

Futuras líneas de investigación deben ir orientadas al estudio de la OCS en: (a) investigaciones longitudinales, para ver si ésta disminuye con el paso de la edad y con el desarrollo de los procesos cognitivos en las distintas etapas evolutivas, (b) incluir instrumentos que midan las variables género y cultura, a fin de poder visualizar y comprender los factores que determinan el comportamiento de la escala en hombres, que es distinta a lo que se ha reportado en investigaciones anteriores, e (c) incluir otras variables psicosociales, que puedan influir en que la OCS sea más elevada en el grupo de 60 años y más, como estrés, vulnerabilidad, amenazas percibidas en el entorno, percepción de riesgo o pérdida y estado funcional en el adulto mayor.

Como posible limitante está la transversalidad del proceso de recolección de datos, siendo necesario profundizar acerca de cómo va cambiando a lo largo de la vida la orientación a la comparación social, concebida no como un patrón de personalidad estable, sino como una predisposición cognitiva modulada por aspectos evolutivos. Una segunda limitación sería no haber tomado otras medidas psicosociales como el género y cultura, para poder explicar de mejor forma los 
hallazgos encontrados en nuestra investigación. Una tercera limitación se debe a que al ser un estudio exploratorio, no se tomaron en cuenta otras variables demográficas, como estado civil, nivel socio - económico, educación y ocupación, para diferenciar a los grupos y ver cómo éstas influyen en la OCS. Y la cuarta limitación sería no haber tomado otras medidas con las cuales correlaciona la OCS y que pueden influir en la tendencia a utilizarla en determinados grupos.

\section{Referencias}

Barrientos, J. (2006). Inicio sexual en el norte chileno: Transición dolorosa hacia una nueva normatividad. Sexualidade, Gênero e Reprodução na Juventude, 28, 1-6.

Buunk, A. P. (2006). Procesos de la comparación social en pacientes que sufren de cáncer. Psicodebate 6, 29 - 44.

Buunk, A. P. (2006). Responses to a happily married other: The role of relationship satisfaction and social comparison orientation. Personal Relationships, 13, $397-409$.

Buunk, A. P., Collins, R., Taylor, S., Van Yperen, N., \& Dakof, G. (1990). The affective consequences of social comparison: Either direction has it ups and downs. Journal of Personality and Social Psychology, 59, $1238-1249$

Buunk, A. P., Belmonte, J., Peiró, J., Zurriaga, R., \& Gibbons, F. (2005). Diferencias individuales en la comparación social: Propiedades de la escala española de orientación hacia la comparación social. Revista Latinoamericana de Psicología, 37, 561 - 579.

Buunk, A. P., \& Brenninkmeijer, V. (2001). When individuals dislike exposure to an actively coping role model: Mood change as related to depression and social comparison orientation. European Journal of Social Psychology, 31, 537 - 548.

Buunk, A. P., \& Mussweiler, T. (2001). New directions in social comparison research. European Journal of Social Psychology, 31, 467 - 475.

Buunk, A. P., Peiró, J.M., Rodríguez, I., \& Bravo, M.J. (2007). A loss of status and a sense of defeat: An evolutionary perspective on professional burnout. European Journal of Personality, 21, 471-485.

Buunk, A. P., Ybema, J., Gibbons, F., \& Ipenburg, M. (2001). Neuroticism and social comparison orientation as moderators of affective responses to social comparison at work. Journal of Personality, 69, $745-763$.

Buunk, A. P., Ybema, J., Van Der Zee, K., Schaufeli, W., \& Gibbons, F. (2001). The affective consequences of social comparison as related to professional burnout and social comparison orientation. European Journal of Social Psychology, 31, 337 - 351.

Buunk, A. P., Zurriaga, R., González - Roma, V., \& Subirats, M. (2003). Engaging in upward and downward comparisons as a determinant of relative deprivation at a work: A longitudinal study. Journal of Vocational Behavior, 62, 370 - 388 .

Buunk, A. P., Zurriaga, R., González, P., Terol, C., \& López - Roig, S. (2006). Targets and dimensions of social comparison among people with spinal cord injury and other health problems. British Journal of Health psychology, 11, $677-693$.

Buunk, A. P., Zurriaga, R., Peiró, J., Nauta, A., \& Gosalvez, I. (2005). Social comparisons at work as related to a cooperative social climate and to individual differences in social comparison orientation. Applied Psychology: An International Review, 54, 61 - 80.

Festinger, L. (1954). A theory of social comparison processes. Human Relations, 7, 117-140.

Frieswijk, N., Buunk, B., Steverink, N., \& Slaets, J. (2004). The interpretation of social comparison and its relation to life satisfaction among elderly people: Does frailty make a difference? Journal of Gerontology: Psychological Sciences, 59B, 250-257.

Gerrard, M., Gibbons, F., Reis - Bergan, M., Trudeau, L., Vande Lune, L., \& Buunk, B. (2002). Inhibitory effects of drinker and nodrinker prototypes on adolescent alcohol consumption. Health Psychology, $21,601-609$.
Gibbons, F., \& Buunk, A. P. (1999). Individual differences in social comparison: Development of a scale of social comparison orientation. Journal of Personality and Social Psychology, 76, 129 - 142.

Gibbons, F.X., Gerrard, M., Lando, H.A., \& McGovern, P.G. (1991). Social comparison and smoking cessation: The role of the "Typical Smoker". Journal of Experimental Social Psychology, 27, 239-258.

Guimond, S., Branscombe, N., Brunot, S., Bunnk, A.P., Chatard, A., Désert, M., García, D., Haque, S., Martinot, D., \& Yzerbyt, V. (2007). Culture, gender, and the self: Variations and impact of social comparison processes. Journal of Personality and Social Psychology, 92, 1118 - 1134.

Guimond, S., Chatard, A., Martinot, D., Crisp, R., \& Redersdorff, S. (2006). Social comparison, self - stereotyping, and gender differences in self construal. Journal of Personality and Social Psychology, 90, 221 - 242.

Gómez - Jacinto, L., (2005). Comparación social y autoevaluación desde un enfoque evolucionista. Escritos de Psicología, 5, 2 - 14.

Heider, F. (1958). The psychology of interpersonal relations. New York: Wiley.

Huerta, J., \& Arduras, A. (2004). Socialización y desarrollo de la motivación: Una perspectiva sociocultural. Porto-Alegre, 1, 11- 37.

Jones, D. (2001). Social comparison and body image: Attractiveness comparisons to model and peers among adolescent girls and boys. Sex Roles, 45, $645-664$

Michinov, E., \& Michinov, N. (2001). The similarity hypothesis: A test of the moderating role of social comparison orientation. European Journal of Social Psychology, 31, 549 - 555.

Michinov, N., (2007). Social comparison and affect: A study among elderly Women. The Journal of Social Psychology, 147, 175 - 189.

Milosavljevic, V., (2007). Estadísticas para la equidad de género. Magnitudes y tendencias en América Latina. Fondo de desarrollo de las Naciones Unidas para las mujeres, Naciones Unidas, Santiago: CEPAL.

Silva, J., \& Barrientos, J. (2008). Guiones sexuales de la seducción, el erotismo y los encuentros sexuales en el norte de Chile. Estudos Feministas, Florianapolis, 16, $539-556$.

Suls, J., Martín, R., \& Wheeler, L. (2002). Social comparison: Why, with, whom, and with what effect. Current Direction in Psychological Science, $11,159-163$.

Suls, J., \& Wills, T. A. (1991). Social comparison: Contemporary theory and research. Hillsdale, NJ: Erlbaum.

Tajfel, H. (1984). Grupos humanos y categorías sociales. Barcelona: Herder.

Taylor, S., \& Lobel, M. (1989). Social comparison activity under threat: Downward evaluation and upward contacts. Psychological Review, $96,569-575$

Taylor, S., Wood, J., \& Lichtman, R. (1985). Social comparison in adjustment to breast cancer. Journal of Personality and Social Psychology, $49,1169-1183$

Thau, S., Aquino, K., \& Wittek, R. (2007). An extension of uncertainty management theory to the self: The relationship between justice, social comparison orientation and antisocial work behaviors. Journal of Applied Psychology, 92, 250-258.

Terol, C., Pons, N., Neipp, M., Rodríguez - Marín, J., Buunk, A.P., Martín - Aragón, M., \& Sánchez, P. (2008). Estrategias de comparación social y resultados en salud: Un estudio de adaptación y evaluación en muestra española de pacientes crónicos. Cuadernos de Medicina Psicosomática y Psiquiatría de Enlace, 84/85, 1 - 13.

Turner, J. C. (1990). Redescubrir el grupo social. Madrid: Ediciones Morata

Urzúa, A., Castillo, P., Gemmel, M., \& Campos, C. (2010). Relación entre la orientación a la comparación social y el auto reporte de la calidad de vida. Revista Colegio de Psicólogos del Perú, 9, 25 - 30.

Van Yperen, N., Brenninkmeijer, V., \& Buunk, B. (2006). People's responses to upward social comparisons: The role of the individual's effort - performance expectancy. British Journal of Social Psychology, $45,519-533$

Van der Zee, K., Buunk, B., \& Sanderman, R. (1996). The relationship between social comparison processes and personality. Personality and Individual Differences, 20, $551-565$. 
Van der Zee, K., Buunk, B., \& Sanderman, R. (1998). Neuroticism and reactions to social comparison information among cancer patients. Journal of Personality, 66, $175-194$.

Van der Zee, K., Buunk, B., Sanderman, R., Botke, G., \& Fons van den Bergh. (2000). Social comparison and coping with cancer treatment. Personality and Individual Differences, 28, 17 - 34.

Van der Zee, K., Oldersma, F., Buunk, B., \& Bos, D. (1998). Social comparison preferences among cancer patients as related to neuroticism and social comparison orientation. Journal of Personality and Social Psychology, 75, $801-810$.

Wheeler, L., \& Miyake, K. (1992). Social comparison in everyday life. Journal of Personality and Social Psychology, 62, 760-773.
Wigfield, A., Eccles, J., Mac Iver, D., Reuman, D., \& Midgley, C. (1991). Transition during early adolescence: Changes in children's domain specific self-perception and general self- esteem across the transition to junior high school, Developmental Psychology, 27, 552 - 565.

Wood, J. (1989). Theory and research concerning social comparison of personal attributes. Psychological Bulletin, 106, 231 - 248.

Ybema, J. \& Buunk, B. (1995). Affective responses to social comparison: A study among disabled individuals. British Journal of Social Psychology, 34, $279-292 . b$.

Ybema, J. \& Buunk, B. P. (1993). Aiming at the top? Upward social comparison of abilities after failure. European Journal of Social Psychology, 23, $627-645$. 